CAHIERS DE

NARRATOLOGIE

\section{Cahiers de Narratologie}

Analyse et théorie narratives

$26 \mid 2014$

Nouvelles frontières du récit. Au-delà de l'opposition entre factuel et fictionnel

\title{
Préface. Nouvelles frontières du récit.
}

Au-delà de l'opposition entre factuel et fictionnel

\section{Alessandro Leiduan}

\section{(2) OpenEdition}

\section{Journals}

Electronic version

URL: http://journals.openedition.org/narratologie/6815

DOI: 10.4000/narratologie.6815

ISSN: $1765-307 X$

Publisher

LIRCES

\section{Electronic reference}

Alessandro Leiduan, « Préface. Nouvelles frontières du récit. », Cahiers de Narratologie [Online], 26।

2014, Online since 11 September 2014, connection on 22 September 2020. URL : http://

journals.openedition.org/narratologie/6815; DOI : https://doi.org/10.4000/narratologie.6815

This text was automatically generated on 22 September 2020.

Article L.111-1 du Code de la propriété intellectuelle. 


\title{
Préface. Nouvelles frontières du récit.
}

\author{
$\mathrm{Au}$-delà de l'opposition entre factuel et fictionnel
}

\section{Alessandro Leiduan}

1 L'une des idées les mieux partagées en narratologie est celle qui ramène la diversité des récits existants à l'opposition que recouvrent les notions de factuel et fictionnel. Il n'est toutefois pas toujours clair ce qui permet d'affilier un récit à l'une ou l'autre de ces catégories. Le critère de discrimination généralement retenu est l'attitude mentale des actants de la communication (producteurs et récepteurs du récit) vis-à-vis de l'histoire racontée: s'ils croient à cette histoire, le récit sera factuel, s'ils font seulement semblant d'y croire, le récit sera fictionnel. Mais, comment être sûr que l'attitude adoptée soit la même, en l'absence d'un critère certain et irréfutable, permettant de discriminer le factuel du fictionnel ? Inférer le statut factuel ou fictionnel d'un récit à partir des indices décelables dans le texte n'est pas toujours chose aisée : faut-il s'appuyer sur la nature réelle ou imaginaire des faits racontés? Ou sur l'apparence textuelle du récit? Ou sur le nom de genre sous lequel le récit décline son identité narrative ? Le choix est lourd de conséquences dans la mesure où, suivant le critère choisi, les résultats peuvent varier du tout au tout. Comment être sûr alors, du statut factuel ou fictionnel d'un récit, si les critères de classification sont tous mutuellement falsifiables?

\section{Approche ontologique}

2 Un premier critère de distinction entre récits factuels et fictionnels identifie comme critère discriminant la nature réelle ou imaginaire de l'histoire racontée. Le problème est qu'aucune histoire n'est entièrement réelle ou imaginaire. Si l'on dépouillait l'ensemble des récits existants à la recherche d'un récit ne faisant état que d'événements réels ou que d'événements imaginaires, on n'en trouverait aucun avec ces caractéristiques. Tous les récits mêlent, en effet, des références réelles et imaginaires et, lorsqu'on en qualifie certains de "factuels» et d'autres de 
«fictionnels», on ne veut pas signifier que leurs contenus respectifs soient intégralement de l'ordre des choses empiriques (pour les récits factuels) ou de l'ordre des choses imaginaires (pour les récits fictionnels) ${ }^{1}$. Les romans, par exemple, accueillent systématiquement dans la trame imaginaire de l'histoire qu'ils racontent, des éléments référentiels, à savoir des faits, des lieux et des personnes que notre encyclopédie enregistre comme "réels " (la Révolution française, la Russie, le Cardinal Richelieu) ${ }^{2}$ et qui ne devraient donc pas trouver place dans ce genre de récit (à entendre de manière rigoureusement littérale l'appellation de «fictionnel » qu'on leur attribue). Quant à la « réalité » dont relèveraient les choses racontées dans un récit factuel, elle n'est pas si empirique qu'on pourrait le penser ${ }^{3}$. La réalité qu'on appelle «fait »a, en effet, ceci de spécifique, qu'elle n'est déjà plus là quand on la raconte, elle ne peut, donc, être reconstituée qu'a posteriori, à partir de ce qui survit d'elle au moment où elle a déjà disparu (les traces) ou à partir de ce que peuvent nous en dire ceux qui ont assisté directement à son déroulement (les témoignages). Et cependant, on ne peut pas s'attendre à ce que traces et témoignages soient, à eux seuls, capables de fournir la trame d'un récit achevé répondant à toutes les questions que l'on pourrait se poser sur la manière dont un fait s'est déroulé, sur les causes qui l'ont provoqué, sur les conséquences qu'il a déclenchées ${ }^{4}$. Les différents éléments qui nous permettent de reconstituer le déroulement d'un fait ne peuvent être vraiment utilisés à cet effet que s'ils sont interprétés, que si leurs parties obscures sont éclairées, leurs lacunes comblées, leurs contradictions levées. De ce travail d'interprétation témoignent les différentes hypothèses que les professionnels des écritures factuelles (historiens, biographes, journalistes, etc.) incorporent dans leurs récits pour éclairer ce qui, dans les versions précédentes de l'histoire qu'ils racontent, résistait aux efforts de compréhension des lecteurs. Le narrateur pourra, par exemple, imaginer un fait dont aucun témoignage n'a jamais fait état (sans que l'on puisse arguer, de cette omission, que le fait en question n'a jamais pu avoir lieu) ou ramener l'agir de tel ou tel autre sujet de l'histoire à une intention déterminée (là aussi, sans se sentir obligé de subordonner la formulation de cette intention à la condition de trouver dans les propos d'un témoin une confirmation explicite de cette conjecture) parvenant ainsi conférer à l'histoire racontée une unité de sens qu'on aurait en vain cherchée dans les versions précédentes de la même histoire (à cause d'un éclairage encore incomplet des non-dits des sources documentaires). Si l'histoire ainsi racontée apparaît plus lisible qu'elle ne l'était auparavant, rien ne garantit pourtant la crédibilité de la parole du Narrateur (surtout si la garantie que l'on recherche est de nature empirique, la seule d'où, en principe, devrait dépendre, selon une interprétation littérale de la notion de «factualité », la crédibilité d'un récit factuel) ${ }^{5}$. Le récit ne pourra donc être tenu pour factuel qu'en faisant l'impasse sur les parties non-factuelles de son contenu. Ce constat s'applique à l'ensemble des récits existants, car, si tous les récits mêlent des références réelles et imaginaires, il n'est possible de les qualifier de « réels» ou d'«imaginaires » qu'en faisant abstraction d'un certain nombre de leurs propriétés sémantiques (celles qui sont en désaccord avec le statut factuel ou fictionnel qu'on leur a attribué).

Ce critère de discrimination est, de surcroît, fragilisé par une méprise grossière : les récits ne mobilisent pas des entités ontologiques ayant la propriété d'être réelles ou imaginaires, mais des entités sémantiques dont l'appartenance à la catégorie des choses réelles ou imaginaires dépend du choix des sujets qui produisent ou interprètent les récits en question de les affilier à l'une ou l'autre de ces catégories. De ce point de vue, l'île au trésor n'est pas moins réelle que la gare de Lyon ${ }^{6}$. Les propriétés qui permettent 
d'affilier la première à la catégorie des référents imaginaires et la deuxième à la catégorie des référents réels ne sont pas inscrites dans ce que ces deux syntagmes signifient, mais dépendent des conventions culturelles qui définissent, dans une société déterminée, ce qui est réel et ce qui est imaginaire. Le sens mobilisé par un récit n'est donc en soi ni réel ni imaginaire, il le devient suivant l'attitude des sujets qui participent à l'acte de communication : s'ils croient à ce que le récit affirme, le récit sera factuel, sinon, il sera fictionnel. Mais ils ne peuvent se déterminer à croire ou à feindre de croire à un récit qu'en faisant abstraction des parties de ce même récit qui contredisent le type de croyance qu'ils ont adoptée. La validité de ce critère de discrimination des récits factuels et fictionnels est donc subordonnée à une condition que les récits existants peinent à satisfaire, eu égard à la nature foncièrement hybride de leur contenu : l'impossibilité de ramener l'intégralité des choses racontées dans un récit, soit à la catégorie des choses réelles, soit à celle des choses imaginaires, invalide toute appréciation du caractère factuel ou fictionnel d'un récit, élaborée à partir d'un critère de discrimination si mal défini.

\section{Approche textuelle}

Un deuxième critère de classification départage les récits factuels et fictionnels suivant l'apparence textuelle du récit. Les lecteurs d'un récit se détermineraient à croire ou à feindre de croire à l'histoire racontée en fonction des caractéristiques formelles et thématiques qui définissent l'identité textuelle du récit en question. Suivant la nature de ces caractéristiques, les destinataires d'un récit seraient enclins à y reconnaître un récit fictionnel ou un récit factuel.

5 Historiquement, la factualité s'est construite sur des bases empiriques, elle s'accommode donc mal de tout ce qui trouve sa source ailleurs que dans l'expérience. Un certain nombre de traits de type thématique ou formel en sont venus, par conséquent, à être proscrits du domaine textuel de la factualité. C'est le cas, sur le plan thématique, des affirmations ayant pour objet des occurrences de l'expérience nonvérifiables (faits possibles, probables, incertains, etc.) ou des affirmations ayant pour objet le psychisme humain (la vie intérieure des autres, mais aussi, dans certains cas ${ }^{7}$, celle du narrateur n'ont pas leur place dans un récit factuel). Sur le plan formel, le même sort a été réservé aux affirmations qui étirent la temporalité de l'histoire racontée en accélérant ou en retardant, par moments, son déroulement, ainsi qu'aux affirmations qui plongent le lecteur dans un état d'incertitude cognitive concernant l'objet du récit (car cela revient à reproduire la logique narrative des fictions qui jouent effectivement à alterner ombre et lumière, opacité et clarté, mystère et intelligibilité pour aguicher et entretenir tout au long du récit l'intérêt du lecteur) ${ }^{8}$.

6 Et cependant, est-ce qu'on a le droit de transformer les régularités observables sur le plan textuel dans le domaine factuel ou fictionnel en normes constitutives de la factualité et de la fictionnalité ? Est-il suffisant, par exemple, de constater que les récits factuels privilégient, sur le plan thématique, l'objectivité à la subjectivité, l'expérience vérifiable (faits certains, avérés) à l'expérience non-vérifiable (faits probables, possibles), pour conclure que tout récit qui oserait explorer le psychisme d'un individu réel ou qui mentionnerait des faits qu'aucune source documentaire ne permet de confirmer, mériterait d'être assigné d'office à la catégorie des récits fictionnels ?9 Devrait-on, par exemple, affilier à la catégorie de la fiction, tous les reportages qui 
usent de certaines techniques de racolage narratif $»^{10}$ dans le but d'entretenir le public dans un état de curiosité ou de suspense, sous prétexte que le recours à ces techniques est monnaie courante dans le domaine fictionnel ${ }^{11}$ ?

7 La possession d'un trait généralement attesté dans le domaine fictionnel ne fait pas automatiquement d'un récit un récit fictionnel. Le même raisonnement s'applique aux caractéristiques textuelles d'un récit factuel : combien de romans déclinent-ils leur contenu dans des formes qui sont le plus souvent attestées dans le domaine factuel? Clarisse de Richardson est un recueil de lettres, Moll Flanders de Defoe, un livre de mémoires, les romans de Balzac, des chroniques... Faut-il les assimiler à de vrais récits factuels seulement parce qu'ils coulent leur contenu dans le moule d'une forme textuelle de type factuel? Auquel cas, il faudrait ranger sous la rubrique des récits historiographiques ou journalistiques un nombre assez considérable de romans d'inspiration réaliste ${ }^{12}$.

8 S'il y a donc des traits sémantiques ou syntaxiques qui ne semblent pas à leur place dans un texte factuel, mais seulement dans un texte fictionnel, ce n'est pas nécessairement parce qu'ils sont absolument incompatibles avec la «factualité » et uniquement compatible avec la «fictionnalité ». Les formes textuelles à travers lesquelles se sont déployées les narrativités factuelles et fictionnelles ne sont pas les seules formes possibles que les écrivains qui ont pratiqué ces types d'écriture auraient pu utiliser : la factualité n'est pas l'otage des conventions historiques qui ont modelé jusqu'ici les discours factuels et la fictionnalité ne l'est pas non plus à l'égard des conventions historiques qui ont modelé jusqu'ici les discours fictionnels ${ }^{13}$.

9 L'erreur de cette approche est, donc, d'hypostasier les traits textuels qui ont caractérisé le déploiement historique de la narration factuelle et fictionnelle au point d'en faire les critères définitoires absolus de toute forme de factualité ou de fictionnalité narrative.

\section{Approche générique (para-textuelle)}

On peut recourir à une troisième approche pour départager les récits factuels et fictionnels: l'élément discriminant, cette fois, est l'indication para-textuelle par laquelle un récit décline son identité générique. Le simple fait de lire roman ou essai historique sur la couverture d'un livre suffit à renseigner le lecteur sur la nature fictionnelle ou factuelle des faits qu'il lira. Mais, est-ce uniquement à partir des informations inscrites dans le sens des mots roman ou essai historique que nous choisissons s'il faut croire ou non à l'histoire racontée ? L'apparence textuelle n'y estelle pour rien dans ce choix ? L'horizon d'attente ${ }^{14}$ (sérieux ou ludique) qu'évoquent pour nous certains noms de genres (roman, reportage, tragédie, comédie, documentaire, chronique, etc.), ne comporte-t-il pas aussi une idée concernant l'apparence textuelle de l'œuvre ${ }^{15} \mathrm{Si}$, par exemple, le régime générique de l'œuvre en question est fictionnel (un roman, un film d'aventure...), on ne s'attendra pas à trouver dans son apparence textuelle des éléments thématiques ou formels qui sont généralement attestés dans un domaine factuel. Et si le régime générique est factuel (une biographie, un documentaire...), on ne s'attendra pas à y trouver des procédés narratifs qui sont communs à la plupart des fictions. La factualité, on le sait, s'accommode mal de la subjectivité, la fictionnalité d'une temporalité comprimée. Mais l'identité textuelle d'un récit est-elle toujours ajustée à son identité générique? Deux cas de figure sont possibles : si elle est ajustée, en clair, s'il y a concordance entre les traits qui définissent 
l'identité textuelle d'un récit et les traits qui définissent son identité générique, alors le récit en question entretiendra avec le genre qui sert à le caractériser une relation d' exemplification ${ }^{16}$ : il possédera, au niveau textuel, les propriétés qui sont inscrites dans le sens du nom qui exprime son identité générique (c'est la situation qui pose le moins de problèmes, car il est aisé de dire s'il s'agit d'un récit factuel ou fictionnel). Mais, que se passe-t-il quand les moyens textuels ne sont pas en adéquation avec les fonctions génériques? L'une des caractéristiques les plus significatives de la narration contemporaine est celle qui consiste à mettre en circulation des récits qui laissent le public dans l'incertitude la plus totale quant à la nature factuelle ou fictionnelle de l'histoire racontée. La raison de cette incertitude est liée à l'emploi de moyens textuels qui semblent peu (ou pas du tout) en adéquation avec les fins qui sont inscrites dans le nom de genre sous lequel les récits en question déclinent leur identité. Que penser, par exemple, d'un reportage qui explorerait la vie intérieure d'un personnage historique sans s'embarrasser d'étayer la description de ses pensées les plus secrètes par l'exhibition de sources fiables attestant que le personnage en question a pu effectivement penser, dans telle ou telle autre circonstance, les choses que le reportage lui attribue? Faut-il ranger le reportage en question dans la catégorie des récits factuels (comme sa dénomination générique nous autoriserait à le faire) ou dans celle des récits fictionnels (comme semblent l'indiquer les moyens textuels employés) ? Et que dire de ces romans qui imitent des formes de narration factuelles sans dire clairement aux lecteurs, par un quelconque indice para-textuel, qu'il s'agit d'une instanciation ludique, non pas sérieuse, de l'acte de communication imité?

11 Faut-il passer outre les incongruités textuelles des œuvres en question et se déterminer à les tenir pour factuelles ou fictionnelles uniquement à partir de leur nom de genre ? La limite de ce critère de discrimination est de croire que l'apparence textuelle d'un récit n'y est pour rien dans la détermination de son statut factuel ou fictionnel, les lecteurs d'un roman ou d'un essai historique (pour ne citer que ces deux exemples) se détermineraient à croire ou à feindre de croire à ce qu'on leur raconte, à partir des instructions que tout nom de genre porte inscrites en lui-même ${ }^{17}$, sans guère se soucier des discordances éventuelles qu'il pourrait y avoir entre lesdites instructions et l'apparence textuelle du récit (ce qui revient à postuler, abusivement, que le rapport de tous les récits au genre dont ils dépendent relèverait de la phénoménologie de l' exemplification).

\section{Critique du mentalisme narratologique}

Prises isolément, les approches qu'on vient de passer en revue échouent, donc, à décrire convenablement les phénomènes narratifs que recouvre l'opposition entre factuel et fictionnel. Ces approches se heurtent, on l'a vu, à l'existence de formes narratives qui mettent en échec les critères d'après lesquels elles prétendent départager les récits factuels et fictionnels. On ne peut se contenter de la typologie narrative à laquelle elles prétendent ramener l'ensemble des récits existants qu'en faisant abstraction de tout ce qui rend les récits en question irréductibles à une typologie de ce genre.

Mais leur défaut majeur est de circonscrire l'étude des effets déclenchés par la lecture d'un récit aux seuls phénomènes qui témoignent de l'attention mentale prêtée par le lecteur à l'histoire qu'on lui raconte. Selon ces trois approches, le statut fictionnel ou 
factuel d'un récit pourrait être déterminé en s'intéressant uniquement à l'attitude mentale des lecteurs vis-à-vis de l'histoire racontée. Or, la réception d'un récit est, certes, une affaire mentale, dans la mesure où, si le sens d'un récit n'est pas compris, son interaction sociale sera pratiquement inexistante. Il est donc indispensable de s'intéresser aux différents paramètres culturels (ontologiques, textuels ou paratextuels) qui permettent à une société de qualifier un contenu sémantique de "réel » ou « imaginaire ». Mais les destinataires d'un récit ne sont pas des êtres désincarnés qui se limiteraient à déchiffrer le sens d'une histoire sans permettre ensuite aux contenus sémantiques ainsi emmagasinés dans leur conscience d'interagir avec les projets, les entreprises, les actions qu'ils pourraient accomplir dans le monde réel. La compréhension d'un récit se prolonge dans l'adoption d'une attitude pratique en adéquation ou non avec la nature de l'histoire qu'on a lue. C'est donc en prenant en compte l'impact des récits sur l'aptitude à agir de la société qu'il conviendra de repenser la signification des catégories factuelles et fictionnelles qui ont servi à départager leurs contenus $^{18}$. Les paramètres de tout type d'action étant réglés sur ce à quoi l'on croit (et non pas sur ce à quoi l'on fait seulement semblant de croire), le fait d'adhérer à une croyance (comme l'exigent les croyances qui trouvent leur source dans un récit factuel) ou de ne pas y adhérer (comme l'exigent les conventions culturelles qui régissent l'attitude de la société vis-à-vis des croyances mobilisées par un récit fictionnel) a pour corollaire d'autoriser certains comportements et d'en interdire d'autres, les croyances licites agissant comme des prescriptions, les croyances illicites comme des interdictions, les unes poussent à l'action, les autres l'interdisent. Les récits factuels et fictionnels exercent donc une fonction régulatrice, non seulement sur les croyances, mais aussi et surtout, sur les conduites sociales. Le problème est que cette fonction est généralement méconnue au profit d'une description simpliste de leurs effets sociaux: l'impact des récits factuels relèverait de la sphère cognitive, celui des récits fictionnels, de la sphère esthétique. On lirait les journaux, les livres d'histoire, les biographies sans autre objectif que celui de se renseigner sur ce qui s'est passé dans le monde et on s'abreuverait de scénarios imaginaires dans le seul but de s'enivrer d'émotions fortes. Or, cette caractérisation est manifestement insuffisante voire même trompeuse : elle couvre plus qu'elle ne dévoile la fonction effective remplie par les récits factuels et fictionnels.

\section{Comment repenser les notions de factuel et de fictionnel en termes non-mentalistes?}

L'existence des récits factuels ne se justifie pas uniquement en regard d'un horizon d'attente qui ne comporterait le moindre calcul des bienfaits escomptables de l'application à la réalité du savoir qu'on peut tirer de ce genre de récits. On ne lit pas un journal, un livre d'histoire, un livre de mémoires dans le seul but de "s'instruire ». On ne saurait donc pas dire que les récits factuels ont pour seule vocation de permettre l'épanouissement intellectuel des lecteurs. Les besoins cognitifs que les récits factuels permettent de satisfaire sont finalisés à l'acquisition de certaines compétences permettant ensuite aux lecteurs d'accomplir efficacement certaines actions dans un environnement social déterminé. Le lecteur qui apprend, à la lecture d'un journal, que la consommation de certains aliments peut nuire à la santé, a la possibilité de modifier ses habitudes alimentaires. Le lecteur qui a appris, dans les livres d'histoire, comment 
des dictatures se sont mises en place, des guerres ont éclaté, des préjugés se sont ancrés dans la mentalité collective condamnant certaines minorités sociales à subir un traitement discriminatoire, a la possibilité, dès qu'il constate dans son environnement politique et social des signes avant-coureurs d'évènements de ce type, de donner l'alarme, en mettant en alerte ses concitoyens sur les dangers que leur communauté est en train de courir, afin que des mesures concrètes soient prises pour éviter le pire. Le savoir mobilisé par les récits factuels mériterait donc d'être requalifié en termes de "savoir-faire ", dans la mesure où il n'est pas stocké dans la mémoire des lecteurs à des fins de contemplation désintéressée, mais qu'il est réinvesti tout de suite dans la réalité sous forme de comportements ou de projets adaptés aux conditions d'exercice de la liberté humaine dans un environnement social déterminé. La performativité est donc l'horizon implicite de toute narrativité factuelle: les informations transmises par ce genre de récits sont transformées en critères d'action définissant ce qu'on peut faire ou ne pas faire dans une société déterminée.

Dans cette même optique, la fictionnalité gagnerait à être redéfinie, non pas seulement comme la forme à travers laquelle se déploie la vie esthétique de la société, mais comme le lieu de dissolution de tous les comportements qui ne sont pas compatibles avec la préservation de l'ordre social existant. Le propre de la fiction est de concourir indirectement à décrédibiliser les comportements sociaux auxquels ses contenus font référence. $\mathrm{Du}$ fait que, par exemple, les lecteurs d'un roman ne peuvent pas croire aux faits représentés dans ce roman, mais uniquement faire semblant d'y croire, il s'ensuit qu'ils n'auront pas envie de réinstancier dans la vie réelle les faits en question, car il s'agit, pour eux, de faits qui ne peuvent trouver place que dans un contexte ludique (celui de la fiction), non pas dans un contexte sérieux (celui de la réalité). Si les formes à travers lesquelles se déploient les comportements observables dans la vie sociale ne ressemblent pas à celles qui sont représentées dans un roman, ce n'est pas nécessairement parce que les comportements romanesques ne pourraient pas être réinstanciés dans la vie réelle, mais parce que la société a été éduquée à croire que ce qui est possible dans le monde fictionnel ne doit pas l'être dans le monde réel. La vie sociale s'est donc déployée à travers des formes qui sont antithétiques à celles qui ont été représentées dans les romans. Les scénarios fictionnels ont ainsi fonctionné comme des anti-modèles à travers lesquels les comportements incompatibles avec les règles de la vie sociale ont été dilués dans les eaux irréelles de la fiction afin d'être mis définitivement hors tout circuit de reproduction sociale ${ }^{19}$. Le mot fiction n'est pas seulement l'antithèse de tout ce que recouvrent des mots comme factuel, référentiel, expérientiel, car la perception sociale de la réalité (à laquelle tous ces mots renvoient) ne serait pas ce qu'elle est, si les œuvres fictionnelles ne contribuaient pas à délimiter les frontières de son extension en dissolvant comme irréel tout ce qui s'écarte des représentations socialement admises. La fiction est donc le présupposé de la réalité, ce sans quoi la réalité serait indiscernable de ce qu'elle n'est pas (l'imaginaire).

Envisagées ainsi, factualité et fictionnalité cessent d'être de simples lieux narratifs où s'expriment les attitudes mentales que la société peut adopter vis-à-vis d'une histoire (l'une relevant de la sphère cognitive, l'autre de la sphère esthétique) pour être enfin reconnues comme des dispositifs qui façonnent, de manière peu visible mais sûre, les comportements observables dans la vie sociale. 


\section{Au-delà de l'opposition entre factuel et fictionnel}

17 Si elle semble convaincante sur le plan théorique, la distinction entre récits factuels et récits fictionnels ne trouve d'ailleurs, comme on l'a vu, que très partiellement confirmation dans l'histoire des genres narratifs, celle-ci étant caractérisée par de nombreux échanges entre l'univers factuel et l'univers fictionnel ${ }^{20}$. Et si, de toute l'histoire des genres narratifs, on ne considère que la page la plus récente, cette distinction apparaît presque entièrement infondée: les récits conventionnellement qualifiés de «factuels» ou de "fictionnels » se déclinent désormais dans des formes textuelles pratiquement indiscernables, invalidant ainsi l'idée selon laquelle à la représentation d'une histoire réelle ou imaginaire correspondraient nécessairement des procédés textuels différents, voire antinomiques. L'hypothèse qui guide ce travail est que la tension entre les traits thématiques et formels des textes et les usages traditionnels auxquels ces mêmes textes semblent destinés de par leur dénomination générique a pour effet d'instituer des usages déviants qui mériteraient d'être reconnus comme tels au lieu d'être ignorés ou dissimulés sous les catégories génériques traditionnelles. Les articles réunis dans ce numéro étudient des textes qui ne remplissent pas complètement les fonctions qui sont inscrites dans la définition de la catégorie générique dont ils relèvent. Le nom de genre par lequel on les désigne est donc inapproprié, car il faudrait les nommer autrement conformément aux fonctions nouvelles qu'ils remplissent. Si le système de classification des genres s'accommode de l'emploi de noms génériques inappropriés, c'est pour pérenniser des usages d'un certain type (les "universaux pragmatiques ») ${ }^{21}$ et pour empêcher l'émergence d'usages concurrentiels (évidemment moins compatibles avec l'idéologie qui préside aux usages traditionnels). Cette inertie classificatoire est donc fonctionnelle à la pérennisation de l'idéologie qui façonne notre attitude à l'égard des choses que nous sommes tenus de considérer comme "réelles » et des choses que nous sommes tenus de considérer comme « irréelles ».

18 François Jost explore l'univers de la feintise cinématographique, c'est-à-dire le champ sémiotique qui s'étend entre la fiction proprement dite et le documentaire, en passant en revue différents types de tromperies audiovisuelles, Alessandro Leiduan étudie, dans le sillage d'Umberto Eco, les dérives factuelles de la fiction, en dégageant la dimension factuelle cachée dans toute interaction entre une œuvre fictionnelle et l'imaginaire social, Pierre-François Peirano s'attache à étudier les différentes manières de relater sur le mode de la fiction un même évènement historique (l'expédition Lewis et Clark), Alexandre Prstojevic analyse le roman Cheval blanc de Lénine ou l'histoire autre de Régine Robin (1979), premier représentant historique d'un (sous)genre littéraire que l'on pourrait appeler récit-hybride, Alexandre Eyriès et Nicolas Pélissier se penchent sur la pratique du journalisme narratif et sur les rapports, complexes et équivoques, qu'elle entretient avec le phénomène du storytelling, Laurent Collet décortique, dans une perspective narratologique, les pratiques qui visent à anticiper l'expérience vécue de l'utilisateur d'un produit/service en cours de conception (scenario-based designed), Elisa Bricco étudie l'imbrication entre factuel et fictionnel dans un corpus de quatre bandes dessinées racontant des événements tragiques de l'Histoire contemporaine, Stéphanie Neu analyse le roman italien contemporain Timira (2013) et teste la valeur heuristique de la notion d'« œuvre métisse », théorisée par le collectif d'écrivain Wu Ming (les 
auteurs du roman), enfin, Maurizio Actis Grosso tisse d'impalpables réseaux affinitaires entre les romans d'un corpus hétérogène, mêlant étroitement histoire et fiction.

\section{NOTES}

1. «Pas plus que le langage n'est le monde, le récit n'est la réalité. Néanmoins, cela ne l'empêche pas d'y faire référence, avec des variations telles que, parfois, à lire ou à voir un récit, nous pensons être dans un monde imaginaire et, à d'autres moments, dans notre monde. Aussi n'est-il pas possible de considérer que « les éléments de la fiction (personnages, temps et lieu, etc.) sont évidemment fictifs » (...). Dans un même récit, certains le sont (superman, Madame Bovary), d'autres pas (New York, Rouen). » F. Jost, Comprendre la télévision, Paris, Colin, 2005, p. 78.

2. C'est par le biais d'un nom de personne réelle (par exemple, Napoléon), d'un toponyme référentiel (la Russie) ou d'un nom relatant un évènement historique (la Révolution française) que le récit fictionnel inscrit dans l'univers représenté des éléments issus du monde référentiel. Que ces « îlots référentiels » (C. Montalbetti) ne fonctionnent pas, dans un récit fictionnel, comme ils fonctionnent dans un récit factuel, c'est probablement vrai, mais, pour neutraliser leurs propriétés référentielles, c'est-à-dire, pour empêcher Napoléon et Russie de signifier le personnage historique et le lieu géographique que l'on connait, il faut que les interprètes des récits fictionnels où apparaissent de tels noms prennent garde de ne pas associer ces mots à tout ce que notre encyclopédie enregistre comme "réel », ce qui prouve que les noms en question ne sont pas naturellement imaginaires et qu'ils sont même plus proches, pour nous, de l'ordre des choses « réelles» que de celui des choses « irréelles ». Sur cette problématique, voir : C. Montalbetti, La fiction, Paris, Flammarion, 2001, pp. 31-36.

3. «Si un laboratoire est un lieu où se déroulent des expériences scientifiques, l'historien est, par définition, un chercheur à qui les expériences, au sens propre du terme, sont interdites. Pour une discipline qui étudie en tant que tels des phénomènes irréversibles dans le temps, il est impossible de reproduire une révolution, un défrichement, un mouvement religieux, non seulement dans la pratique, mais aussi dans le principe. Cette caractéristique n'est pas le propre de la seule historiographie - il suffit de penser à l'astrophysique et à la paléontologie "C. Ginzburg, «Preuves et possibilités » dans Le fil et les traces, Paris, Verdier, 2010, pp. 448-449. Une réalité, pourrait-on dire, en synthétisant le point de vue de C. Ginzburg, est empirique si elle peut être vérifiée, si on peut la mettre à l'épreuve de l'expérience, si elle peut être sujette à des jugements de vérité ou de fausseté. Or, la doxa narratologique considère que cette possibilité est une prérogative des récits factuels : «Les récits référentiels sont vérifiables et incomplets, alors que les récits non référentiels sont invérifiables et complets ». D. Cohn, « La fiction : une mise au point » dans Le propre de la fiction, Paris, Seuil, 2001, p. 33.

4. Si tel était vraiment le cas, il n'y aurait point besoin d'entreprendre à nouveau le récit du fait en question, on pourrait très bien se contenter de la version livrée par les différents témoins ou de celle que tout un chacun serait en mesure de reconstruire s'il pouvait avoir accès à toutes les sources documentaires qui permettent aux historiens, aux journalistes, aux biographes et aux autres spécialistes des écritures factuelles de redonner vie, par leurs récits, aux vestiges d'une histoire passée.

5. D'autant plus qu'émettre une hypothèse pour rendre compte d'un point obscur d'une histoire réelle ou concevoir de toutes pièces l'intrigue d'un roman sont deux opérations qui semblent 
différentes, mais qui obéissent, en réalité, aux mêmes contraintes formelles : ne pas enfreindre les principes qui définissent ce qu'une société considère comme réel (ou comme pouvant l'être). Bien qu'elles soient accomplies à des fins différents, les deux opérations aboutissent donc à un même résultat : un récit vraisemblable, tirant sa légitimité, non pas de son adéquation à la réalité empirique, mais de sa conformité au système d'expectatives qui règlent la perception sociale de ce qu'on appelle la "réalité ». Celle-ci n'est pas incompatible avec un décryptage nonempirique, à condition, bien entendu, que le recours à l'imagination se fasse sous le contrôle du vraisemblable, du plausible, de l'opinable, c'est-à-dire de l'ensemble des choses qu'une société est disposée à croire comme pouvant avoir lieu (même si, dans les faits, ces choses n'ont jamais eu lieu ou qu'elles ont eu lieu quand personne ne pouvait les observer).

6. Voir, à ce sujet, l'entrée «Référence » dans O. Ducrot et J.-M. Schaeffer, Nouveau dictionnaire encyclopédique des sciences du langage, Paris, Seuil, 1995, p. 360.

7. Je pense notamment aux écritures factuelles de type journalistique, historiographique, biographique.

8. Les règles qui président à l'articulation narrative de la factualité s'accommodent mal d'un type d'écriture qui mettrait trop de temps à raconter comment les choses se sont réellement passées. «Le récepteur de ce genre de récit, écrit justement Raphaël Baroni, ne se complaît pas dans l'attente incertaine de la fin de l'histoire et dans l'indétermination du sens ", il exige du narrateur qu'il en vienne au fait sans trop tergiverser. Cf. R. Baroni, L'œuvre du temps, Paris, Seuil, 2009 , p. 59.

9. L'exploration de la vie intérieure des personnages d'un récit est (ou, en tout cas, devrait être) une prérogative des seuls récits fictionnels. Comme l'explique Dorrit Cohn, « cette radioscopie [du psychisme humain] fait appel à des procédés - entre autres le discours indirect libre auxquels des narrateurs se proposant de construire des représentations référentielles (non fictionnelles) ne peuvent avoir accès ». D. Cohn, op. cit., p. 33 (c'est moi qui souligne).

10. Par « racolage narratif», nous n'entendons pas nécessairement quelque chose de négatif: l'intégration d'éléments fictionnels dans un récit factuel ne répond pas toujours, en effet, au besoin de rendre plus attrayante l'histoire racontée, mais elle vise, parfois, à accroître la capacité de ladite histoire de nous éclairer sur la manière dont s'est probablement déroulée une certaine succession de faits. Ainsi, la micro-histoire, le docu-fiction, le roman-enquête nous éclairent sur la manière dont se sont déroulés certains faits que les livres d'histoire, les documentaires et les enquêtes de la police n'ont pas su entièrement élucider. La fiction, ici, a vocation à combler les lacunes du discours historique, documentaire et judiciaire. Voir, à ce sujet, l'entretien que j'ai réalisé avec $\mathrm{R}$. Baroni et qui a été publié, sous le titre de «La narratologie à l'épreuve du panfictionnalisme », dans la revue Modèles linguistiques. URL : http://ml.revues.org/244. Consulté le 31.05.2014.

11. «Forme parasite» de la factualité (J.-L. Austin), la fiction est devenue, au fil du temps, le "dépotoir " de tous les traits thématiques et formels que les formes de discours sérieux ne reconnaissent pas comme propres (on pourrait la caractériser comme l'équivalent symbolique, dans le domaine de la narratologie textuelle, de l'inconscient freudien). C'est, en effet, dans les récits fictionnels qu'ont trouvé refuge les éléments textuels qui ne pouvaient pas trouver place dans une œuvre destinée à un usage factuel. En tant que « parasite » des récits factuels, la fiction se nourrit de ce que les auteurs de récits factuels considèrent comme impropre à l'usage. Contraints par l'idéologie qui gouverne leur écriture à privilégier l'objectivité et à s'interdire de « doper » l'attrait de l'histoire qu'ils racontent par des effets de « racolage narratif » (suspense, curiosité, surprise) qui semblent incompatibles avec les finalités sociales d'un discours sérieux, les professionnels des écritures factuelles ont ainsi contribué indirectement à cantonner dans le domaine de la fiction tout ce qui permet au récit de devenir le lieu où la subjectivité du narrateur entre en résonance avec celle de ses lecteurs, où s'exprime l'aspiration humaine vers des mondes possibles différents des mondes existants, où l'on cherche des réponses aux questions que le sens 
émanant des discours sérieux ne parvient pas à résoudre. Sur la fiction et le mensonge envisagés comme « actes parasites », voir de J.-L. Austin, «Feindre » dans Ecrits philosophiques, Seuil, 1994, pp. 206-228.

12. Il en va de même, dans le domaine de l'énonciation télévisuelle, pour les séries qui imitent les procédés de filmage et de montage des reportages. "Produite par l'agence de presse Capa, la série Police District (diffusée à raison de 4 à 6 épisodes entre 2000 et 2003 sur M6), (...) mime le reportage d'aujourd'hui grâce à divers procédés de filmage ou de montage : dutch angle, flou, bougé, filé, décadrage, très gros-plan, profondeur de champ très accentuée, éblouissement, effets stroboscopiques, tout cela enchaîné à vive allure. Toujours sur l'épaule, la caméra de Police District confère à l'image une indéniable humanité, celle du cameraman cette fois-ci.» F. Jost, op. cit., p. 83.

13. J.-M. Schaeffer a montré que tous les indices de fictionnalité répertoriés par des auteurs comme K. Hamburger ou A. Banfield ne sont pas une prérogative des seuls récits fictionnels, mais sont également présents dans les récits factuels. Voir, à ce sujet, J.-M. Schaeffer, Pourquoi la fiction?, Paris, Seuil, 1999, pp. 264-265.

14. Pour cette notion, voir : H.-R. Jauss, Pour une herméneutique littéraire, Paris, Gallimard, 1988.

15. Jauss définit, justement, l'horizon d'attente comme un « ensemble de règles préexistant pour orienter la compréhension du lecteur et lui permettre une réception appréciative », cité par $\mathrm{M}$. Macé, Le genre littéraire, Paris, Flammarion, 2004, p. 22.

16. Pour cette notion, voir: N. Goodman, Languages of Art, Indianapolis, Hackett, 1976.

17. Lire « roman » revient à recevoir une instruction du type : « tu n'as pas le droit de croire à ce que tu liras, car rien n'est vraiment arrivé, tout a été inventé ». «Essai historique » contient, en revanche, l'instruction suivante : « tout ce que tu lis s'offre à toi comme vrai, il t'appartient donc de mettre à l'épreuve sa vérité en la comparant à celle qui se dégage de tous les autres discours sérieux qui ont été écrits sur le sujet ».

18. Ai-je le droit de croire à ce que ce texte me raconte? Ai-je le droit de faire interagir le sens de ce texte avec le sens que je prête aux choses réelles? Ai-je le droit de modifier ma conduite dans le monde réel à partir du sens mobilisé par ce texte ? Ai-je le droit de m'attendre à ce que les autres aussi, à la lecture de ce texte, acceptent de modifier leur propre conduite ? C'est à partir de ces questions qu'il faudrait commencer à départager l'identité factuelle ou fictionnelle d'un récit, en évitant de circonscrire leurs effets à la sphère cognitive ou à la sphère esthétique. Si le texte admet une réponse active (un comportement), il relèvera de la catégorie du factuel. S'il n'admet qu'une réponse passive (un non-comportement), il relèvera de la catégorie du fictionnel.

19. De ce point de vue, on pourrait dire que le « donquichottisme » ou le «bovarysme » sont des constructions idéologiques qui occultent la vraie nature de l'influence de la fiction sur la société en présentant comme "réelles" des conséquences sociales qui ne sont qu' «imaginaires » : la perte du sens de la réalité causé par la lecture des romans n'est, en effet, attestée que... dans les romans. L'un des fils conducteurs thématiques du livre de J.-M. Schaeffer sur la fiction est d'ailleurs une prise de position polémique à l'égard de l'une des premières expressions historiques de l'idéologie du "donquichottisme": la théorie platonicienne de l'art. Cf. J.-M. Schaeffer, Pourquoi la fiction? op. cit.

20. Voir les nombreux exemples cités par Marielle Macé dans l'Introduction à son livre sur le genre littéraire. Cf. M. Macé, op. cit., pp. 13-46.

21. Les « universaux pragmatiques » sont, selon J.-M. Schaeffer, les situations-type auxquelles on pourrait ramener toute forme de communication. Ces situations-type peuvent être définies à partir des marqueurs textuels et des index para-textuels d'une œuvre : il existe ainsi des marqueurs textuels de la fiction (par exemple, la description directe des pensées et états psychologiques d'un tiers) et des index para-textuels (par exemple, l'indication générique : roman). Lorsque les marqueurs textuels ne sont pas en accord avec les marqueurs para-textuels, la relation d'un texte avec le genre dont il dépend relève de la phénoménologie de la modulation. Or, selon Schaeffer, la 
modulation n'affecterait pas l'usage social d'un texte : s'ils ne sont pas en adéquation avec l'usage codifié, les traits sémantiques ou syntaxiques d'un texte n'instituent pas un usage d'un type nouveau, mais ils se limitent à rendre plus opaque ou indéterminé l'usage traditionnel. Il y aurait, selon lui, des universaux pragmatiques structurant l'usage social des actes de communication : la non-concordance entre les prescriptions qui régissent l'usage social d'un texte et les moyens (thématiques et formels) mis en œuvre pour satisfaire à ces prescriptions ne donnerait pas lieu à la naissance d'usages nouveaux. La société ne saurait adopter vis-à-vis du sens mobilisé par un texte que les attitudes pragmatiques consacrées par la tradition. Aucun autre usage ne peut être conçu qui ne soit pas conforme aux usages qui existent déjà. "Je pense qu'on peut avancer que les noms de genres qui ont pour référents des propriétés de l'acte communicationnel sont contextuellement stables, cela dans la mesure où ils se réfèrent à des universaux pragmatiques, ou du moins à des propriétés dont la stabilité est telle que, comparées à d'autres traits génériques extrêmement variables en contexte, on peut les traiter comme des traits invariants. » J.-M. Schaeffer, Qu'est-ce qu'un genre littéraire ? Paris, Seuil, 1989, p. 182. 